8. STELLAR AND EXTRAGALACTIC JETS 


\title{
Theory of Stellar and Extragalactic Jets
}

\author{
R. D. Blandford \\ Theoretical Astrophysics 130-33 Callfornia Institute of Technology Pasadena, CA 91125 U.S.A.
}

\begin{abstract}
Different types of astrophysical jets are briefly described and the wide differences in their observed properties are emphasized. Various physical mechanisms that have been proposed for creating jets, based on gas dynamical, magnetic, and radiative stresses are briefly reviewed. It is argued that magnetic flelds are probably generally important for collimating astrophysical jets.
\end{abstract}

\section{Introduction}

Over the past ten years, it has become apparent that jets (i.e., collimated outflows of gas from a compact object) are created under diverse conditions. These jets are confidently interpreted as "exhausts" for the gravitational engines in active galactic nuclei, proto-stars, binary X-ray gources, etc. However, there is still no single example of an astrophysical jet whose energization and collimation we understand. Instead what we have are incomplete theories that identify the primary collimating agent as gas pressure, radiation pressure, or magnetic stress. What is striking about the observations is that jets are observed in objects where gas, radiation, and magnetic stresses have each been inferred to dominate. This suggests that finely-tuned physical conditions are not essential to make jets; all that is necessary is a gravitational potential well and the accretion of orbiting gas.

In this report, I will summarize the observational position contrasting the available diagnostics and observed properties of stellar and extragalactic jets. I shall pay special attention to $\mathrm{SS} 433$ as it is for this source that the strongest case can be made for radiatively-driven jets. SS433 also poses the best defined challenges to the theorist. I shall then go on to give a brief outline of some basic ideas in gas, magnetic, and radiation models and describe some recent developments. See ref. [1] for a more complete discussion of theoretical ideas containing an extensive bibliography. Throughout the text I shall suggest some problems that provide tractable challenges to numerical hydrodynamicists and whose solution would sharpen our intuition about radiation hydrodynamics and may even help explain SS433 and other sources.

\section{Astrophysical Jets}

\section{a) Extragalactic Radio Sources}

The most extensively studied class of jets is associated with extragalactic radio sources [2]. As these are reviewed elsewhere by Perley and Norman in these proceedings, it suffices to distinguish four subclasses and summarize the inferences that can be drawn from the observations regarding the collimation mechanism. 


\section{(i) Powerful Radio Galaxies}

Here the jets are belleved to be highly supersonic as indicated by the hot spots at their ends, and at least mildly relativistic in order to explain the large jet : counterjet flux ratios and the persistence of this one-sidedness on the VLBI scale. (Several observers have argued that jets are intrinsically one-sided [3], but it is this reviewer's belief that only relativistic beaming can account for the observed one-sidedness and that counterjets will generally be found as observations are made with improved dynamic range.) VLBI observations of compact radio sources [5] often show a core-jet morphology, of which the core is interpreted as the self-absorbed inner jet. Polarization observations of compact radio sources imply the absence of thermal plasma and suggest that the jet fluid comprises electrons and positrons. Earlier claims of extensive depolarization in extended jets have been repudiated and it now appears likely that these jets are extremely light and possibly even positronic (Perley, these proceedings).

Early attempts to explain jet collimation through nozzles formed in a spinning gas cloud [4] fail because the jets are now known from VLBI observations to be collimated on scales $\leqslant 1$ pe and the necessary gas pressure would, again, lead to an X-ray luminosity in excess of the observed value. Collimation by an ion pressure-supported cloud surrounding a massive central black hole remains a viable option however. As we discuss further below, one briefly popular model for jet collimation invoking the presence of funnels in a radiation-supported torus surrounding a massive black hole is quite inappropriate to most radio galaxies. This is because the minimum energy required to create the radio source places a lower bound on the mass of the hole. If a radiation-supported torus is present, a luminosity in excess of the Eddington value is expected to leak away. Assuming (conservatively) a total efficiency factor of 0.1 , the nuclear luminosity of a radio galaxy (expected to emerge in the optical and ultraviolet regions of the spectrum) is

$$
L_{\text {nucl }} \gtrsim 10^{45}\left(U_{\min } / 10^{60} \mathrm{erg}\right) \mathrm{erg} \mathrm{s}^{-1} \text {. }
$$

This greatly exceeds the observed luminosity in most of the more energetic radio galaxies.

In many jets, the minimum internal pressure derived from the equipartition argument, exceeds the maximum external gas pressure estimated from X-ray observations by an order of magnitude. This has been taken to indicate that magnetic stresses have an important role in confining the jets and, by extension, are largely responsible for creating them.

\section{(ii) Weak Radio Galaxies}

The jets associated with weak radio galaxies are, by contrast, generally believed to be sub- or trans-sonic flows because most of the radio power in a weak source originates fairly close to the associated galactic nuclei and because the more distant regions exhibit irregular morphology. It is possible that most of the jets in these sources are re-collimated on scales $\sim 100 \mathrm{pc}-1 \mathrm{kpc}$, perhaps through nozzles formed in a gas cloud [6]. However, as with the powerful radio galaxies. the jets associated with weak sources are extremely unlikely to be collimated by radiation pressure.

\section{(iii) Quasars}

The jets associated with quasars show some unique morphological characteristics. They are nearly all one-sided and far more radio luminous than the jets associated with comparably strong radio galaxies. Their internal pressures are even harder to confine by surrounding gas alone [7]. The one-sidedness is again commonly attributed to relativistic beaming and the enhanced dissipation rate to interaction of the jet with an inhomogeneous circum-quasar medium. However, many quasars are sufficiently luminous in the optical and UV to satisfy equation (1) and so their jets could be created by radiation pressure. (A subset of quasars contain superluminally expanding compact radio sources and, as we shall see later, these are hard to create by radiation pressure. 


\section{(iv) Seyfert Galaxies}

Seyfert galaxies are relatively under-luminous as radio sources. Typically, the ratio of their radio to optical powers is $\approx 10^{-5}$. not so different trom the "radio-quiet" quasars. Nevertheless, many of them are close enough to us that they can be detected and their radio structures resolved [B]. Many Seyfert radio sources appear to contain short ( $\$ 1 \mathrm{kpc}$ ) jets. However, they are so weak that there are no direct observational limits on their collimation mechanism.

\section{b) Galactic Center}

Our own galactic center contains an unusually intense radio source [20] almost coincident with an infrared source and the dynamical center of a conspicuous distribution of gas clouds [21]. There is a radio source elongated perpendicular to the galactic plane which has been interpreted as a pair of jets [22].

\section{c) Stellar Jets}

\section{(i) Young Stars}

As this topic is reviewed by Mundt in these proceedings, it is only necessary to repeat three simple conclusions:

(1) The outflows are continuous rather than impulsive just as was found to be true of the extragalactic sources.

(2) Radiation pressure is inadequate to drive the jets and bipolar outflows as the momentum in the stellar radiation field, is much less than that inferred in the flows typically by a factor $\approx 100$.

(3) Gas pressure collimation close to the star or proto-star is hard to sustain because the relevant cooling times are short compared with the dynamical timescales.

Several authors have concluded that magnetic flelds are important in collimating these outflows [9]. [10], [11], [12].

\section{(ii) $\mathbf{S \$ 3 3}$}

The observations of this object have been comprehensively summarized [13]. Three sets of emission lines, two of which arise in outflowing jets precessing with a $164 \mathrm{~d}$ period on a cone with vertex angle $20^{\circ}$. whose axis is inclined at an angle $79^{\circ}$ to the line of sight. The jet speed $(0.26 \mathrm{c})$ is surprisingly stable (to better than one percent) even allowing for jitter in the jet direction. This "kinematical" model is confirmed by radio observations. The jets are collimated within $\$ 2^{a}$. The third set of emission lines shows photometric and spectroscopic periods which are now unambiguously interpreted as binary orbital motions with a period of $13.1 \mathrm{~d}$. The mass function is $11 M_{0}$ which allows low mass and high mass solutions. However, if we also use the apparent duration of the secondary eclipse and interpret the Balmer lines as originating partly from a hot spot, then low mass solutions are untenable [14]. The best fit primary mass is $\approx 25 M_{\odot}$ and the associated mass for the (presumed) compact secondary is $\approx 10 M_{\odot}$ thereby ruling out a neutron star identification and suggesting that it is a black hole.

"Nodding" periods of $6.3 \mathrm{~d}$ and $5.8 \mathrm{~d}$ have also been detected in the high velocity system line data. These were anticipated on the hypothesis that the jets were launched perpendicular to a large disk or ring orbiting around the compact secondary star and perturbed by the primary so that they undergo retrograde precession. Neither the amplitude of these motions nor the reason for the inclination of the disk/ring to the orbital plane are properly understood. Many authors have suggested that the primary star has a misaligned spin axis which precesses because of a torque exerted by the secondary and that the "disk" is "slaved" to it [13]. It should be noted however that the observed rate of precession is too rapid for this to be the case for a normal corotating star flling its Roche lobe. 
If we allow for $\sim B$ magnitudes of visual extinction, the observed bolometric luminosity of the system is $\approx 4 \times 10^{38} \mathrm{erg} \mathrm{s}^{-1}$, just below the Eddington limit for a $\approx 10 M_{\odot}$ black hole. However, the mechanical power in the jets may well be much larger. There are two methods for estimating the power. Firstly we can estimate the thrust necessary to inflate the protruberances found at either end of the surrounding radio source, W50. The second method uses the hydrogen recombination rate to compute the mean number of Balmer photons emitted per hydrogen atom on the outflow timescale and normalises this to the observed luminosity of the moving Balmer lines. Both estimates are model-dependent, although it seems hard to avoid the conclusion that the jet power exceeds the Eddington limit for $\left\langle 10 M_{\odot}\right.$ black hole $\sim 10^{38} \mathrm{erg} \mathrm{s}^{-1}$.

In summary, the observations give strong support to an extended kinematical model in which jets are launched with a carefully tuned speed of $0.26 \mathrm{c}$ perpendicular to a precessing disk carrying a strongly super-Eddington mass flux. Radiation pressure is obviously important in SS433 although it is not clear that this alone can produce the tight collimation that we infer. The constancy of the observed speed poses a clear, quantitative challenge to theorists.

\section{(iii) Sco X-1}

It has long been known that the high latitude luminous galactic X-ray binary, Sco X-1, is identified with a triple radio source [15]. Just as is the case with the extragalactic radio sources, the central component is positionally coincident with the X-ray (and optical) source. The two outer components, found at distances of $\sim 1 p c$ on opposite sides of the central source, are unusually compact. The minimum internal pressures in these outer components greatly exceed the ambient interstellar pressure. They are also observed to be moving very slowly $v=35 \mathrm{kms}^{-1}$ in the case of the NE component. This effectively rules out ram pressure confinement and suggests that the hot spots are foci where only a small fraction of the bulk kinetic energy is dissipated [16]. This interpretation is supported by the recent discovery of an outer pair of radio components collinear with the previously known three [17].

Again, magnetic and gas pressure focusing have to be invoked, but as Sco $\mathrm{X}-1$ is a fairly luminous source $\left(L_{*} \sim 10^{37} \mathrm{erg} \mathrm{s}^{-1}\right)$ it is possible that the outflow is launched by radiation pressure.

\section{(iv) Cyg X-3}

One of the more reliably observed emanations from $\mathrm{Cyg} \mathrm{X}-3$ is intense, highly variable radio emission. Recent VLBI observations have resolved the source and shown that it is elongated and appears to be expanding at a speed $\sim 9 / 4$ [19]. The similarity to early VLBI observations of extragalactic sources encourages the belief that this source too will eventually be shown to contain tightly collimated jets. It would be of considerable interest to determine if the outflows inferred in other types of compact binary systems (e.g. RW Sext, [1B]) are bipolar.

\section{(v) $\mathrm{CH}$ Cyg/R Aqu}

The symbiotic stars $\mathrm{CH}$ Cyg and $\mathrm{R}$ Aqu exhibit linear radio features that have been interpreted as jets (Taylor and Seaquist, these proceedings). An expansion speed in excess of $3000 \mathrm{~km} \mathrm{~s}^{-1}$ and the presence of a white dwarf accreting at or near the Eddington limit has been inferred in the former case. 


\section{d) Supernova Remnants}

\section{(i) Bilateral Remnants}

A new class of supernova remnants has been proposed [23]. These "bilateral" remnants are characterized by a fan shape with a compact source at the vertex. Sinuous features emanating from the compact source have been seen. These may also be jets.

\section{(ii) Crab Nebula}

In 1970, Van den Bergh discovered a feature protuding from the northern rim of the Crab Nebula which he called a jet. More recent studies have found a coincident radio feature [24] and shown that it is expanding transversely with a speed $\sim 400 \mathrm{kms}^{-1}$ [25]. Various authors have suggested that this jet originates from the central pulsar [26].

\section{Thermal Pressure-Driven Jets}

We now consider some of the ways by which jets may be energised and collimated starting with mechanisms which rely mainly on gas pressure to bring this about.

\section{a) Nozzles}

An early suggestion for the collimation of jets [4] was that light, high entropy gas is continuously created in the center of a spinning cloud

of denser gas. This fluid escapes along a pair of channels aligned with the rotation axis. Application of Bernoulli's equation tells us that the flow becomes trans-sonic when the external pressure falls to roughly half the stagnation pressure of the jet flow. The cross sectional area of a steady, one-dimensional jet will adjust so that the jet pressure matches the external pressure and it will be minimized at the sonic point. This is a De Laval nozzle (Fig, 1).

To give a simple illustration, suppose we have a radiation-dominated fluid with an ultrarelativistic internal sound speed $\left(3^{-x_{C}}\right)$. The relativistic Bernoulli equation relates the pressure $p$, to the baryon proper density $n$ and the Lorentz factor of the jet bulk velocity $\gamma$

$$
p \propto n / \gamma
$$

where we have ignored the rest mass of the baryons in computing the relativistic enthalpy. The equation of state is $p \propto n^{4 / 3}$ and so solving, we obtain

$$
p=p_{0} \gamma^{-4}
$$

where $p_{0}$ is the stagnation pressure in the jet. The cross sectional area $A \propto n^{-1}\left(\gamma^{2}-1\right)^{-1 / 2} \propto \gamma^{3}\left(\gamma^{2}-1\right)^{-1 / 2}$ is indeed minimized when $\gamma=(3 / 2)^{1 / 2}$ (i.e., the jet speed is $\left.3-k_{c}\right)$ and $p=(4 / 9) p_{0}$.

Numerical simulations [27] indicate that this mechanism can operate if the power in the jet is matched to the pressure $p_{\text {ext }}$ and the scale height $h$ of the surrounding cloud. If the jet power is too low, a series of bubbles rising subsonically under buoyancy forces is produced. If the jet is too powerful, the cloud is completely disrupted.

The trans-sonic point is a critical point of the flow and a special condition must be satisfied there if the jet speed is to become supersonic. Critical points are a general feature of stationary adiabatic flows and arise wherever the flow speed equals a relevant wave speed. They can be thought of as relics of the initial value problem. Transients associated with the establishment of the flow will die away everywhere except at the sonic points where backward propagating waves are trapped. If the flow speed is not large compared with the escape velocity, then the gravitational acceleration must also be included. A critical point condition must still be satisfied but the cross-sectional area need no longer be minimized there. This is generally true of spherical wind 


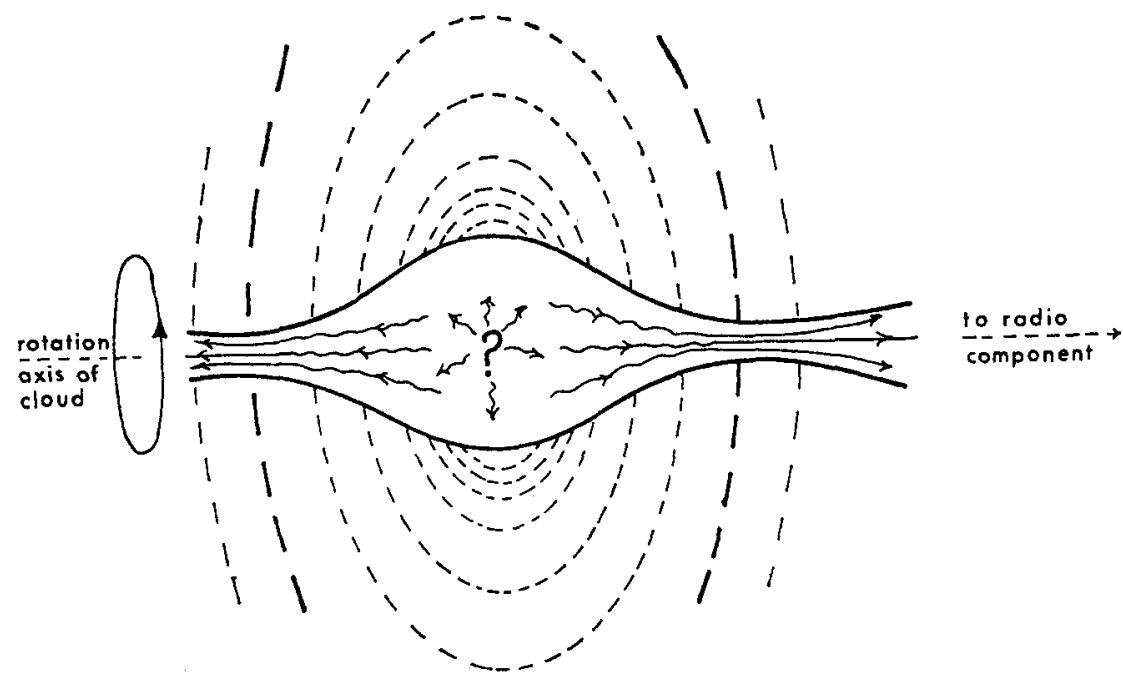

Figure 1. Hypothesised production of jets by the nozzle mechanism. A light fluid (e.g. relativistically hot or radiation-dominated plasma) is copiously produced at the center of a spinning gas cloud whose isobars are denoted by dashed contours. It is envisaged that a stationary flow be established along the spin axis, and that this flow becomes supersonic when the gas pressure has fallen to roughly half the central stagnation pressure. Note that it is the symmetry axis of the confining gas cloud rather than that of the source of the plasma which ultimately defines the direction of the jets.

solutions (e.g., Lucy, these proceedings).

\section{b) Thermally Driven Finds}

The X-rays radiated by a quasar may illuminate and heat the outer parts of an orbiting accretion disk [28]. The gas will then be heated to the Compton temperature $\langle h \bar{\nu} / 4 k$, where $\bar{\nu}$ is the intensity-weighted mean frequency of the radiation fleld) so that its sound speed exceeds the escape velocity. Most of the incident radiation should then be converted into kinetic energy of an escaping wind. This wind would normally flow quasispherically but if some supplementary collimating process (e.g., hydromagnetic torques as discussed below) is present, then this may provide a mechanism for creating slow speed jets. It is of particular interest in the context of Seyfert galaxies (cf. section 2). A complete description of this flow poses a very interesting problem in numerical radiation hydrodynamics as the flow must adjust its optical depth self consistently. (For an alternative description of radiative ablation see ref. [29].)

\section{Hydromagnetic Jets}

As we have argued, there are good reasons for believing that many types of jets are hydromagnetically collimated. Many authors have devised models in which the flelds are purely poloidal and used to separate inflowing gas at low latitudes from high latitude, bipolar outflow. However, all of the observed examples of jets are believed to be spinning and angular momentum is generally acknowledged to be important. This allows a far more efficient collimation mechanism in which "hoop" stresses associated with toroidal fields wrapped around the jet pinch the outflowing gas and create jets [6]. [30]. This toroidal magnetic field can exist both in the jet itself and in the surrounding gas. Indeed, it is necessary that the return current flow at large perpendicular distances from the jet if the magnetic stresses are to confine a jet pressure that is substantially larger than the surrounding gas pressure (cf. 82. Fig. 2). Polarization observations may be able 
to detect toroidal magnetic feld through Faraday rotation (unless the jet and its immediate surroundings contain electrons and positrons rather than thermal plasma).

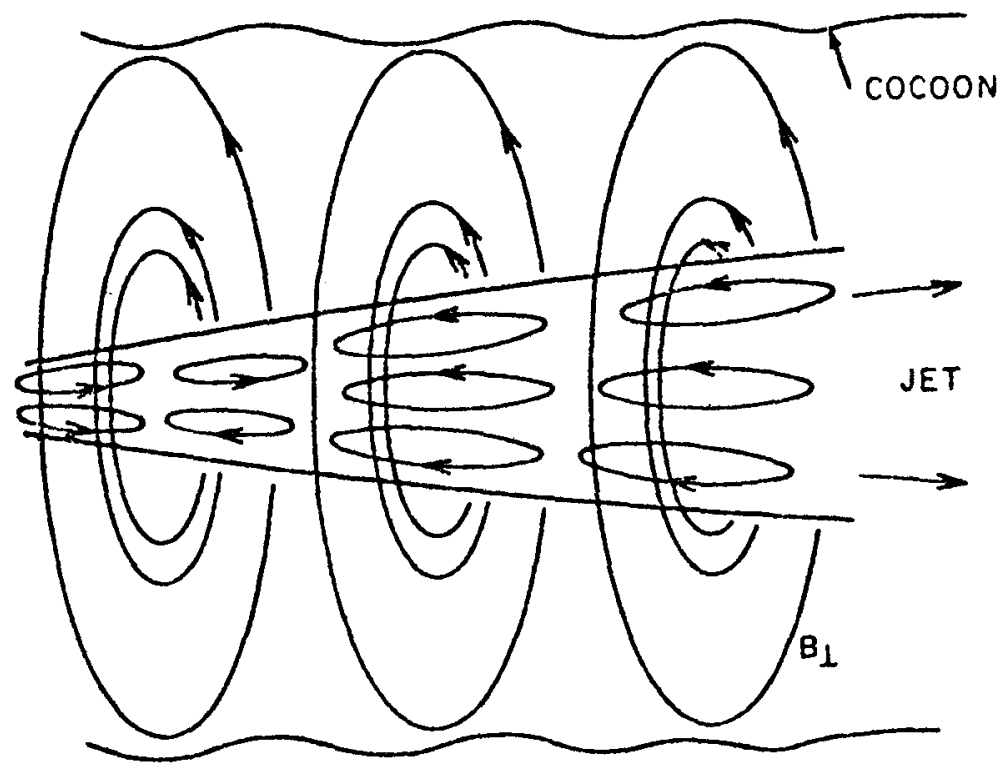

Figure 2. Collimation of a jet by a toroidal magnetic field. In this mechanism, a current flowing parallel to the jet supports a toroidal magnetic field in the surrounding gas which pinches the jet and focusses it. The return current must flow at large distances trom the jet if the confining magnetic pressure is to exceed the ambient external gas pressure. If, as is likely to be the case, there is velocity shear then the magnetic field within the jet may be preferentially stretched out along the axis as is observed to be the case in the more powerful radio jets.

Norman (these proceedings) summarizes recent numerical hydrodynamical work on the propagation of adiabatic jets through unmagnetized circumgalactic gas. It will be of considerable interest to repeat these calculations for magnetically confined jets. Current indications are that magnetically confined jets will have similar stability properties to gas pressure confined jets at least as far as axisymmetric $(m=0)$ and $k i n k(m=1)$ modes are concerned. Shearing $(m=2)$ modes may however be suppresssed in magnetically confined jets by the magnetic tension [32].

We have argued in section 2 that magnetic fields are also likely to be involved in the initial jet production. One mechanism by which this may be brought about occurs when poloidal fields are frozen into a rotating (accretion or circumstellar) disk. In this case a wind can be driven away from the surface of the disk by centrifugal force. We may think of the field lines as fiexible wires and the plasma as beads which slide along them. In the simple example of a poloidal magnetic field line making an angle $\varphi$ with a Keplerian disk, the centrifugal force along the wire is $n^{2} r \cos \varphi$ which cancels the gravitational force to lowest order. To the next order it can easily be shown that the equilibrium of disk particles is unstable and they will be flung out when $\varphi \leq 80^{\circ}$ [17]. [33]. If the disk is partially supported by radical pressure gradients, then a gas must be driven outward by thermal pressure close to the disk before centrifugal force can overcome gravity. When a centrifugal wind is launched from the two disk surfaces in this manner, then the gas will flow outwards until its inertia bends the magnetic field lines backwards along the toroidal direction. As the gas flows further away it will pass through an Alfven point and the magnetic field will become increasingly toroidal and will start to focus the flow towards the rotation axis [33], [34]. 
This mechanism provides a natural way of removing the angular momentum liberated by the infalling gas in an accretion disk. This is particularly important in the case of an isolated disk in a galactic nucleus where there is otherwise no efficient sink for the angular momentum. (There is not necessarily a problem with accretion disks in binary systems, because angular momentum can be transported radially onwards through the disk to be returned to the orbital motion.) Hydromagnetic braking of accretion disks is well suited to study by axisymmetric accretion disks.

Hydromagnetic jet acceleration in a pre-existing funnel has also been considered [35]. In addition, the powering of winds and bipolar outflows by hydromagnetic waves is disussed here by Opher.

A variant on this mechanism may also operate near black holes (Fig. 3). If the black hole is spinning rapidly, then field lines that thread its horizon and which are supported by external toroidal currents can exert a torque on the hole and can extract some of the stored spin energy in an electromagnetic form useful for creating non-thermal emission. In principle, all of the spin energy can be extracted in this manner, though in practice a fraction $\$ 0.3$ will be released. The remainder of the spin energy is absorbed in increasing the irreducible mass of the hole [36], [37], [38]. The spinning black hole can be thought of as a unipolar inductor with an internal resistance $R \sim 100 \Omega$. This roughly matches the impedance of the electromagnetic load in the circuit, the impedance of free space (377n) times a geometrical factor.

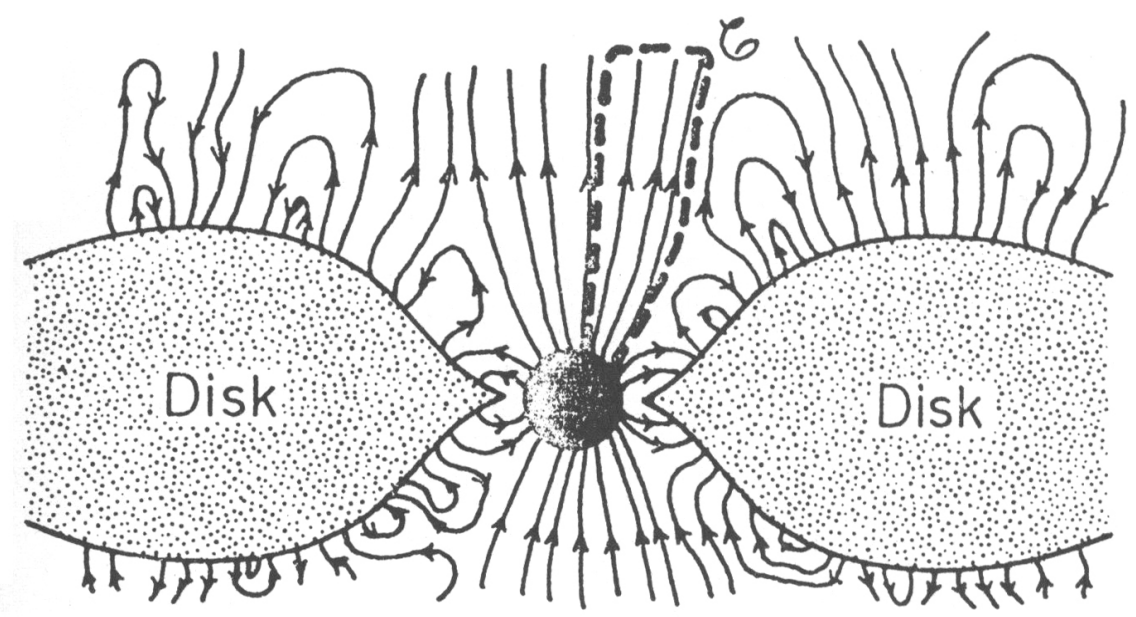

Figure 3. Schematic representation of electromagnetic extraction of energy and angular momentum from a spinning black hole surrounded by an orbitting accretion disk. disk gas may flow out along open field lines leaving the disk surface. The inertia of this gas will cause the field lines to lag with respect to the disk and thereby exert a hydromagnetic torque. Field lines that thread the event horizon of the spinning black hole (generated by currents flowing in the disk) can also carry current There will be an e.m.f, around a circuit threading the hole, such as that shown with dashed lines, and this means that power can be extracted if there is an electrical resistive load on this circuit outside the hole.

As with the MHD wind launched by the disk, the power is almost entirely in the form of electromagnetic Poynting flux. The current is probably carried by electrons and positrons. Electron-positron jets with ultra-relativistic outflow speeds are subject to strong radiative deceleration in a galactic nucleus through inverse Compton scattering of the ambient radiation field. This is avoided when the outflowing power is dominated by electromagnetic Poynting flux [39]. 
For a massive black hole in a galactic nucleus the EMF generated is related to the specific angular momentum, $a$ measured in geometrical units and the magnetic field strength $B$ by

$$
V \sim 10^{19}\left(\frac{B}{10^{4} G}\right)\left(\frac{a}{10^{13} \mathrm{~cm}}\right) \text { Volts . }
$$

The associated power $\left(\sim V^{2} / R, \sim 10^{49}\left(V / 10^{19} \mathrm{Volts}\right)^{2} \mathrm{ergs}^{-1}\right)$ can be quite adequate to power the most powerful extended radio sources.

The origin of these hypothesised magnetic fields poses an intriguing problem in (nonstandard) dynamo theory. It is encouraging that the usual requirements of differential rotation and cyclonic convection will be present in an accretion disk and efficient dynamo action seems hard to avoid.

\section{Radiation Driven Jets}

When the power liberated by an accreting black hole approaches or exceeds the Eddington limit, radiation pressure becomes important. If the accreting gas has angular momentum then it will probably form a thick accretion disk in orbit about the black hole (cf. Hawley, these proceedings). Several authors have suggested that two anti-parallel jets driven by radiation pressure along the spin axis will naturally form under these conditions.

\section{a) $\$ \$ 433$}

As we described above, a good case can be made for the jets in SS433 being created under conditions of radiation dominance. The simplest explanation for obtaining the special outflow speed of $0.26 \mathrm{c}$ is that it originates from line locking [42]. [43].

Suppose that there is a pronounced jump in the continuum of $\mathrm{SS} 433$ beyond the Lyman limit. Then hydrogen (or hydrogenic ions) can be accelerated by Lyman alpha resonance line radiation pressure as long as the comoving wavelength of the resonant photons does not exceed the Lyman limit. If the acceleration occurs sufficiently far from the continuum source that the radiation field is essentially one-dimensional, then the maximum speed, $\beta$, to which the gas can be accelerated is given by

$$
\left(\frac{1+\beta}{1-\beta}\right)^{1 / 2}=\frac{4}{3}
$$

or $\beta=0.28$. This is tantalizingly close to the observed value of $\beta=0.26$.

Unfortunately, there are substantial problems involved in making this mechanism work. The first concerns the minimum area of the continuum source. The continuum luminosity must be sufficient to accelerate the jet $\left(L>M \beta c^{2)}\right.$. As the effective temperature is likely to be $\approx 10^{4} \mathrm{~K}$, this then implies that the source size should exceed $\sim 10^{12} \mathrm{~cm}$. The jet acceleration and collimation must then (for the most plausible case of hydrogen line-locking) occur at a distance $22 \times 10^{13} \mathrm{~cm}$ well outside the binary orbit. Secondly, independent of whether or not the radiation is beamed, the energy flux must exceed the Eddington value (based on Thomson opacity). As the opacity in the Lyman continuum where the edge is formed must greatly exceed the Thomson value it is hard to see how an atmosphere could be sustained. Thirdly, efficient acceleration requires a high degree of clumping in the outflowing gas, and this in turn implies a large overpressure which must be confined.

An alternative class of explanations of the observed constant jet speed in SS433 is based on the following thought experiment. Suppose that we release a test particle above an infinite surface that radiates with uniform density $I_{\nu}$. If the particle scatters the radiation (e.g., by the Thomson process) it will be accelerated away from the plane until, asymptotically, it acquires the velocity of the center of momentum frame of the radiation field $(\beta)$. In other words, in the (primed) frame comoving with the electron

$$
\int_{0}^{\infty} I_{\nu^{\prime}}\left(\nu^{\prime}, \mu^{\prime}\right) \mu^{\prime} d \mu^{\prime} d \nu^{\prime}=0
$$


where $\cos ^{-1} \mu^{\prime}$ is the angle a ray makes with the outward normal. Transforming into the frame of the surface, we see that this condition becomes

$$
\int_{0}^{1} \alpha \mu(1-\beta \mu)(\mu-\beta) d \mu=0 .
$$

Equation ( 7 ) has the solution $\beta=0.45$. Of course, a value of $\beta=0.26$ could be contrived by limb brightening the emission or changing the shape of the surface. It has been suggested $\{[44],[45]$; cf. also Icke and Euldenick, these proceedings) that gas accelerated away from the surface of an accretion disk might form high speed jets in this manner.

However, several complicating factors must be addressed before such a mechanism can be regarded as viable. Firstly gravitational centrifugal forces must be included in solving for the motion of the particle [46]. Secondly the structure of the radiating surface must be considered. Suppose it is a disk of thickness $h$ and the outflow is accelerated from a radius $r$. If the acceleration to speed $\beta$ is to be accomplished within a vertical distance $s r$ (in order to validate the ID approximation) then the radiation flux $F$ must satisfy

$$
F \gtrsim \beta^{2} c^{3} / \kappa r
$$

where $\alpha$ is the relevant opacity (presumably Thomson). If we assume that the surface of the disk has the same opacity as the outflow (and it may be larger), then the radiation force per gram exceeds the surface gravity by a factor $\left(\left(\beta / \beta_{e}\right)^{2}(r / h)\right.$ where $\beta_{a} c$ is the local escape velocity. This expression is necessarily much larger than unity and so it hard to see how the radiating surface can be kept in hydrostatic equilibrium. In other words, it is difficult for a constant radiative flux to accelerate gas smoothly out of a potential well whose gradient is increasing with distance. Thirdly, the mass flux rates that are envisaged suggest that the outflow is optically thick to Thomson scattering near its origin. The radiation field must be determined self-consistently by radiative transfer calculations in the flow and not specified externally.

\section{b) Radiative Acceleration in a Funnel}

Several authors, following LYNDEN-BELL [47], have hypothesized that black holes in active galactic nuclei and X-ray binaries might be surrounded by thick accretion disks and that the funnels defined by these disks might collimate the observed jets. The original idea was that narrow and essentially rigid funnels should be defined by the balance of gravitational, centrifugal and pressure forces. The funnel walls were supposed to radiate Eddington fluxes $c g_{\mathrm{g}} / \kappa_{\mathrm{eg}}$ locally as determined by the local effective gravity $g_{e}$. The total luminosity in the funnel would then be so large that plasma would be accelerated to ultra-relativistic speeds along the funnels and thus account for superluminal radio sources, etc.

This is no longer believed. One reason for this is that as mentioned above thick accretion disks with narrow funnels are believed to be dynamically unstable. Nevertheless, some of the other objections that were raised are of more general interest.

One problem is that the radiation field within the funnel is fairly isotropic because most of the photons will be scattered several times off the funnel walls before they escape. This implies that any outflowing plasma can only be accelerated to a mildly relativistic outflow velocity [48], [49]. It appears that the jet Lorentz factor is limited to $\sim 1.5$ and that most of the acceleration comes trom the top of the funnel, as discussed in the poster by Nobilli et al. (However, a jet of electrons and positrons can be accelerated to a somewhat greater speed.) The most thorough studies of this type of model [50], [51] decompose the radiation field into its first three moments and write down special relativistic fluid equations for radiation-driven outfow in the onedimensional approximation. Even this simple case, ignoring angular momentum, magnetic fields, etc., has a surprisingly rich critical point structure and multiple solutions with the same starting conditions are possible. (The study of critical point structure in genuinely two dimensional flows promises to be a fascinating research problem in formal hydrodynamic theory that will probabll only be properly understood with the aid of numerical simulations.) Indeed, the very notion of a critical point has to be modified when radiative transport is included. When both the radiation pressure and the gas pressure are important, the fluid possesses an adiabatic sound speed and an isothermal sound speed, the former involving the total pressure and the latter just the gas pressure. If radiation transfer terms are included in the equation of motion it turns out that the 
flow has to pass through an isothermal critical point and only near to an adiabatic point [52]. In physical language, we are saying that adiabatic transients trapped near critical point will be damped away by radiative diffusion.

Another problem is that although the normal component of the radiation pressure force can be balanced at the funnel walls, the inevitable tangential component is unbelanced and will probably lead to a significant outfiow of matter [53]. This motivates the discussion of optically thick outflows. The equations of motion for adiabatic radiation-dominated flow in a funnel in a Schwarzschild metric have been written down [54]. I If we measure the mass $m$ of the hole in geometrical units and use the notation of section 3 then these are

$$
\begin{aligned}
& n A \gamma \beta(1-2 m / r)^{1 / 2}=\text { const. } \\
& \left(\mu_{p}+4 p / n\right) \gamma(1-2 m / r)=\text { const } \\
& p / n^{4 / 3}=\text { const. }
\end{aligned}
$$

( $\mu_{p}$ is the proton mass). In contrast to the De Laval nozzle mechanism discussed in section 3 , the pressure in the flow must adjust to a prescribed cross-sectional area $A$ rather than vice versa. In addition, as there is a gravitational field present, the area $A$ need not pass through a minimum here. Any outflow that becomes supersonic must pass through a critical point where its Mach number is unity. (The asymptotic velocity is roughly twice the velocity at the critical point.)

If we use these equations to try to explain the observed speed, $\beta=0.26$, in SS433, we conclude that the critical point must be located at $50-100 \mathrm{~m}$, for a funnel of reasonable shape. If the discharge is $10^{20} \dot{M}_{20} g s^{-1}$ and the black hole has a mass $\sim 10 M_{\odot}$ then the inferred pressure at the critical point is $\sim 2 \times 10^{15} \dot{M}_{R 0}$ dyne $\mathrm{cm}^{-2}$. This exceeds the gas pressure by a factor $\sim 10^{3} \dot{M}_{20}^{-1 / 4}$ and so the assumption that the flow is radiation dominated is vindicated. The optical depth across the jet is $\sim 10^{3} \dot{M}_{20}$ justifying the fluid approximation. However, the effective temperature at the critical point is $\sim 3 \times 10^{7} \dot{M}_{20}^{1 / 4} \mathrm{~K}$. This is not much less than the temperature at which hydrogen burns. The pressure within the surrounding torus would be even higher and so there is the possibility that the any jets that were collimated through a funnel (other problems notwithstanding) would mainly comprise helium. However it is principally the hydrogen by which we observe these jets.

A further difficulty is, once again, that the emergent jet is not very well collimated. A rough lower bound on the opening angle is $0.3 \dot{M}_{20}^{1 / 4}$ (Begelman, private communication). Again, in the case of SS433 at least, some supplementary collimation mechanism seems called for even if radiation pressure is responsible for launching the jets.

\section{c) Numerical Approach}

Although the above analyses of analytically tractable limiting cases have been rather discouraging it still seems reasonable that, as originally sketched by SHAKURA and SUNYAEV [55], supercritical accretion onto a black hole should create bipolar outflow. Numerical computations are clearly required and to date. I only know of one such calculation that includes gravity, angular momentum, radiative transfer viscosity and two-dimensional poloidal flow albeit in a highly simplified manner. This is described in a recent preprint by Eggum, Coroniti, and Katz (Fig. 4). Their treatment is Newtonian and treats the radiation transport by flux-limited diffusion. The torque responsible for angular momentum transport is assumed to be proportional to the radial angular velocity gradient. It therefore ignores the strong shear in the outflowing jet.

In spite of (or possibly because of) these simplifying assumptions, some interesting features of the flow emerge. Large convection cells are established in the accretion disk which inhibit the accretion. High latitude accretion defines a pair of funnels along which two low power jets $\left(L \sim 3 \times 10^{-3} L_{\mathrm{Bdd}}\right)$ escape. The escape speed is $\beta \sim 0.3$ but the collimation is poor. It is to be hoped that future simulations will determine the sensitivity of these results to the specific assumptions that have been made. 

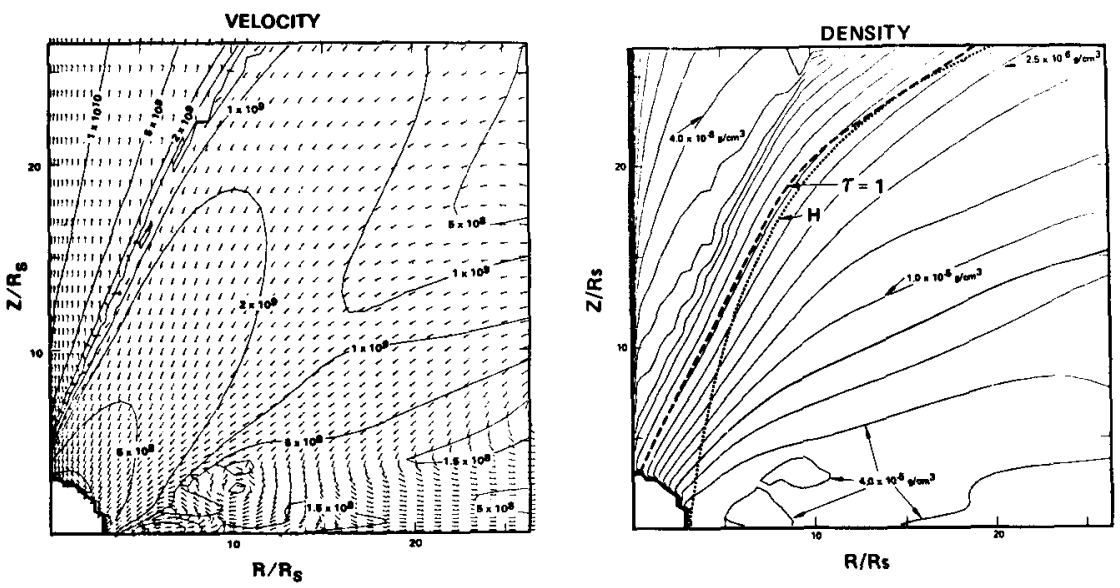

Figure 4. Radiative hydrodynamical calculations carried out by Eggum, Coroniti and Katz (preprint). A super-Eddington accretion flow onto a black hole (approximated using Newtonian gravity) is established. The radiative transport is treated using flux-limited diffusion. A pair of poorly collimated channels are defined by the high latitude accretion. The outflow speed in the jets is $\beta=0.3$ although the mass discharge in the jets is only $\sim 10^{-3}$ of the total accretion rate. The diagram on the left shows the velocity field in the $r-z$ plane using both vectors and contours. Note the strong circulation in the disk. The diagram on the right hand side shows the associated density contours. Note the position of the photosphere $\tau=1$.

\section{Concluding Remarks}

Although this is meant to be a meeting on radiation hydrodynamics, I contend that radiation pressure is probably not generally responsible for the collimation of astrophysical jets. Only in the cases of SS433 and the radio quasars does radiation appear to be strong enough to drive the outflow and here there is no convincing mechanism to focus the jets using photons alone. In the case of the superluminal quasars, there is the additional problem that radiation pressure cannot even produce the inferred outflow speeds.

As has been argued on many occasions in the past, magnetic fields generated by dynamo action in the ubiquitous accretion disks seem to provide the most natural coupling between deep gravitational potential wells and the associated jets. The same magnetic field lines should be spun around the jet by the orbitting disk to become increasingly toroidal at high altitudes and thereby collimate the jets by their pinching action. Unfortunately, the simplest model problems that might be able to demonstrate that this coupling is effective and fairly general are already beyond the scope of analytical methods. The most pressing need seems to be the development of efficient axisymmetric MHD codes to extend the existing gas dynamical and radiation hydrodynamical numerical experiments alluded to above. I suspect that this is true not just of jets but of the majority of astrophysical flows. I hope that the next IAU Colloquium on this topic will legitimately be entitled "Radiation Magnetohydrodynamics in Stars and Compact Objects".

\section{Acknowledgements}

Financial support by the National Science Foundation (AST-84-15355) and the Max Planck Institut für Astrophysik at Garching is gratefully acknowledged. I thank the director of the Institute of Astronomy at Cambridge for hospitality during the writing of this review. I also thank John Hawley and Sterl Phinney for comments on the manuscript and Ferd Coroniti for supplying Fig. 4. 


\section{References}

1. M. C. Begelman, R. D. Blandford, and M. J. Rees: Rev. Mod, Phys. 56, 255 (1984)

2. A. H. Bridle and R. A. Perley: Ann. Rev. Astron. Astrophys. 22.319 (1984)

3. L. Rudnick: "Extragalactic Radio Sources", IAU Symposium No. 97, ed. Heeschen and Wade (Reidel, Dordrecht, Holland 1982), p. 47

4. R. D. Blandford and M. J. Rees: Mon. Not. R. astr. Soc. 169. 395 (1974)

5. K. I. Kellermann and G. Setti (ed.): "VLBI and Compact Radio Sources", IAU Symposium No. 110 (Reidel, Dordrecht, Holland 1984)

8. K. L. Chan and R. N. Henriksen: Ap. J. 241, 534 (1980)

7. R. I Potash and J. F. C. Wardle: Astron. J. B4, 707 (1979)

B. A. S. Wilson: "Extragalactic Radio Sources", IAU Symposium No. 97, ed. Heeschen and Wade (Reidel, Dordrecht, Holland 1982), p. 179

9. A. Königl: Ap. J, 261, 115 (1982)

10. B. Draine: Ap. J. 270, 519 (1983)

11. R. E. Pudritz and C. A. Norman: Ap. J. 274.677 (1983)

12. Y. Uchida and K. Shibata: P.A.S.J. 36, 105 (1984)

13. B. Margon: Ann. Rev. Astron. Astrophys. 22.507 (1984)

14. E. M. Leibowitz: Mon. Not. R. astr. Soc. 210, 279 (1985)

15. E. B. Fomalont, B. J. Geldzahler, R. M. Hjellming, and C. M. Wade: Ap. J. 275, 802 (1983)

16. A. Achterberg, R. D. Blandford, and P. Goldreich: Nature 904, 607 (1983).

17. T. Velusamy, A. Pramesh Rao, and S. Sukumar: Mon. Not. R. astr. Soc. 213, 735 (1985)

18. J. L. Greenstein and J. B. Oke: Ap. J. 258, 209 (1982)

19. B. J. Geldzahier et al.: "VLBI and Compact Radio Sources", IAU Symposium No. 110 (Reidel, Dordrecht, Holland 1984), p. 281.

20. K. Y. Lo et al.: Nature 315, 124 (1985)

21. M. K. Crawford et al.: Nature 315. 467 (1985)

22. K. Y. Lo et al.: Nature 315, 124 (1985)

23. R. M. Becker and D. J. Helfand: Nature 313. 115 (1985)

24. T. Velusamy: Nature 308. 251 (1984)

25. P. Shull, U. Carsentz, M. Sareander, and T. Neckel: Ap. J. Lett. 285, L75 (1984)

26. G. Benford: Ap. J. 2B2. 154 (1984)

27. M. L. Norman, L. L. Smarr, K.-H. A. Winkler, and M. D. Smith: Ap. J. 247. 52 (1981)

28. M. C. Begelman, C. F. McKee, and G. A. Shields: Ap. J. 271, 70 (1983). 
29. A. Königl: Ap. J. 284, 303 (1984)

30. G. Benford: Mon. Not. R. astr. Soc. 183, 29 (1978)

31. H. Cohn: Ap. J, 269, 500 (1983)

32. M. D. Smith and C. A. Norman: Mon. Not. R. astr. Soc. 194, 771 (1981)

33. R. D. Blandford and D. G. Payne: Mon. Not. R. astr. Soc. 199. 883 (1982)

34. J. Sakurai: Astron. Astrophys., in press (1985)

35. J. Fukue: P.A.S.J. 35. 539 (1983)

36. R. D. Blandford and R. L. Znajek: Mon. Not. R. astr. Soc, 179, 433 (1977)

37. D. Macdonald and K. S. Thorne: Mon. Not. R. astron. Soc. 198, 345 (1982)

38. E. S. Phinney: unpublished thesis, University of Cambridge (1983)

39. M. J. Rees: "VLBI and Compact Radio Sources", IAU Symposium No. 110 (Reidel, Dordrecht, Holland 1984)

40. J. C. B. Papaloizou and J. E. Pringle: Mon. Not. R. astr. Soc. 208. 721 (1984)

41. J. C. B. Papaloizou and J. E. Pringle: Mon. Not. R. astr. Soc. 213, 799 (1985)

42. M. Milgrom: Astron. Astrophys. 7B. L9 (1979)

43. P. R. Shapiro, M. Milgrom, and M. J. Rees: preprint (1985)

44. V. Icke: Nature 266, 699 (1977)

45. J. I. Katz: Ap. J. 236, L127 (1980)

46. V. Icke: Astron, J. 85. 329 (1980)

47. D. Lynden-Bell: Phys. Sci. 17, 185 (1978)

48. T. Piran: Ap. J. Lett. 257, L23 (1982)

49. M. Sikora and D. B. Wilson: Mon. Not. R. astr. Soc. 197, 529 (1981)

50. L. Nobilli, M. Calvani, and R. Turolla: Mon. Not. R. astr. Soc. 214, 161 (1985)

51. A. Ferrari, E. Trussoni, R. Rosner, and K. Tsinganos: Astrophys. J. Lett. 277, L35 (1984)

52. R. A. Flammang: Mon. Not. R. astr. Soc. 200. 589 (1984)

53. R. Narayan, R. Nityananda, and P. J. Wiita: Mon. Not. R. astr. Soc. 205, 1103 (1983)

54. J. Fukue: P.A.S.J. 34. 163 (1982)

55. N. I. Shakura and R. A. Sunyaev; Astron. Astrophys. 24, 337 (1973)

\section{Discussion Following Paper Presented by R. D. Blandford}

MCCRAY: If there is a supercritical torus in SS433, is there a problem in hiding the Eddington luminosity $\left(H_{H^{\sim}}-10\right.$ solar masses, $L \sim 10^{99} \mathrm{erg} \mathrm{s}^{-1}$ of the torus? 
BLANDFORD: Not if it comes out in the UV.

NORDLUND: Has the precession of Kerr black holes in external gravitational fields been considered in the case of SS433? What about precession due to non-parallel angular momentum of a Kerr black hole and other external forces (infalling material, magnetic fields)?

BLANDFORD: Yes, there are discussions of Lense-Thirring and geodetic precession in the context of SS433 (e.g. by Martin and Rees and other authors cited in Margon's review). Unfortunately, the predicted orbital period is measured in minutes rather than $13 \mathrm{~d}$ as is observed.

ICKE: Regarding your remark that Eddington-limited thick disks are non-starters as candidates for radio galaxies, doesn't that depend on assuming that the Eddington luminosity is not reprocessed to come out as another form of energy, e.g. mechanical energy?

BLANDFORD: Yes, it does. However, any such reprocessing has to be very efficient. For example, the radio lobes in Centaurus $A$ have a minimum internal energy $\sim 10^{80} \mathrm{erg}$ implying that the black hole have a mass in excess of $10^{7}$ solar masses if we assume a 10 per cent efficiency. The Eddington limit would then exceed $\sim 10^{45} \mathrm{erg} \mathrm{s}^{-1}$ which is a hundred times the central bolometric luminosity.

MICHEL: If, indeed, one has a black hole plus some sort of disk, then this seems a fairly specific model for an AGN. What is the major stumbling block in using these elements to give an interesting model?

BLANDFORD: The major problem in understanding accretion disks theoretically is our ignorance of the viscosity. (See Pringle's 1981 review article for a discussion.)

BOYLE: Since it is possible for a black hole to accrete at any rate (due to photon diffusion rate < infall speed), then surely one cannot just say that the accretion rate is proportional to the observed luminosity.

BLANDFORD: Yes, that's right. Models of spherical accretion allow the radiative efficiency to be arbitrarily low. However, if it is lower than say 10 per cent, then the known bolometric output of distant quasars implies even larger black hole masses than the $10^{8}$ solar masses usually invoked. The question then becomes "where are they now?". A quantitative calculation addressing these issues can be tound in Soltan (1982,MN 200,115). We hope that Space Telescope will find or limit the central masses in many more galaxles.

KROLIK: What is the strongest argument supporting the claim that quasars radiate at a rate near Eddington?

BLANDFORD: The best observational arguments I can offer you for the existence of the very thick rather than thin accretion disks expected when the radiation flux approaches the Eddington limit are:

(i) Most quasars are steady at optical and X-ray frequencies on timescales comparable with the mass of the hole. This suggests the hole is hidden behind a thick cloud of gas.

(ii) The optical spectrum is not a power law and exhibits at least one pronounced bump suggesting that a large fraction of the total luminosity is thermalised over a substantial surface area.

(iii) Most quasars have low polarisation ( $\$ / 2 \%$ ) suggesting that if they have an electron scattering atmosphere then it is not flattened.

None of these arguments is compelling.

PENG: How do you think about our Galactic center? Is the massive black hole theory useful for our Galactic center? According to a recent Annual Review, its luminosity $\sim 10^{\gamma} \mathrm{L}_{\odot}$ is produced by a cluster of massive $\mathrm{OB}$ stars and the central black hole is only $\sim 100 \mathrm{M}_{\odot}$

BLANDFORD: There is excellent evidence that $\mathrm{Sgr} \mathrm{A}^{*}$ is a unique place in the galaxy and good dynamical evidence for a few million solar masses being located there. The hypothesis that this 
is mainly the mass of a black hole is only an interpretation and the alternative view that it be due to a star cluster cannot be ruled out. The idea that there be a black hole mass of $\sim 100 M_{\odot}$ derives from one interpretation of the gamma ray observations.

MEYER: I would like to call attention to the work by T. Sakurai performed during his recent visit to the Max Planck Institute at Garching. Sakurai computed stationary, rotationally symmetric outflows either magnetically or also thermally driven above escape speed in a self-consistent manner, especially also solving the lateral pressure - magnetic field equilibrium perpendicular to the flow direction. His solutions show that all these flows from a rotating source that contain magnetic flux change from a more or less radial structure in the inner regions to a perfectly rotation-axis aligned jet (in two opposite directions). The angular direction alignment occurs essentially on a logarithmic radial scale, thus focussing more and more even the originally closely equatorial flow into the axial direction as more decades in distance are covered. 